I. LUX

I. PAZSIT

\title{
COLLISION DENSITY APPROACH OF RADIATION DAMAGE IN A MULTI-SPECIES MEDIUM
}

Hungarian Academy of Sciences

CENTRAL

RESEARCH

INSTITUTE FOR

PHYSICS 


\title{
COLLISION DENSITY APPROACH OF RADIATION DAMAGE IN A
} MULTI-SPECIES MEDIUM

\author{
I. Lux and I. Pázsit \\ Central Research Institute for Physics \\ H-1525 Budapest 114 , P.O.B.49, Hungary
}

Submitted to Annals of Nuclear Energy 


\section{ABSTRACT}

Space-energy dependent forward type equations for the collision densities of energetic atoms in multi-species semi-infinite homogeneous medium are formulated. Introduction of the one-dimensional isotropic forward-backward model of Fermi for the scattering and application of the Laplace transform with respect to the lethargy variable will lead to a linear differential equation system with constant coefficients. This equation system is solved for an arbitrary number of species and relations between the collision densities and defect distributions of the different species are given in the Kinchin Pease model. The case of an alien particle incident on a two-component target is examined in some detail and the sputtering spectra are given numerically.

\section{АННОТАЦИЯ}

для описания плотности столкновений энергетических атомов в однородном многокомпонентном полупространстве выведены уравнения прямого типа, зависящие от пространства и энергии. Введение одномерной изотропной модели рассеяния вперед-назад типа Ферми и применение трансформации Лапласа по переменной летаргии приводит к системе линейных дифференциальных уравнений с постоянным коэффициентом. Система уравнений решается для произвольного числа компонентов и дается взаимосвязь между плотностьо столкновений и распределением дефектов различного типа в модели радиационного повреждения Кинчин-Пис. Подробно изучается случай попадания в двухкомпонентный материал третьей частицы и для всех трех типов атомов дается спектр распыления в цифровой форме.

\section{KIVONAT}

Tér- és energiafüggơ, elôre tipusu egyenleteket állitunk fel homogén többkomponensủ féltérben energetikus atomok ütközési sürüségére. A Fermiféle izotróp előre-hátra szórási modell bevezetése és a letargiaváltozó szerinti Laplace-transzformáció alkalmazása konstans együtthatóju lineáris differenciálegyenletrendszerre vezet. Az egyenletrendszert tetszôleges számu komponensre megoldjuk, és megadjuk az összefüggést az ütközési sürüségek és a különbözõ defektek között a Kinchin-Pease sugárkárosodási modellben. Részletesen vizsgáljuk kétkomponensü anyagra beesõ harmadik tipusu részecske esetét, és a porlódási spektrumot mindhárom tipusu atomra numerikusan megadjuk. 
1. INTRODUCTION

Relevant quantities of radiation damage / defect distributions, energy deposition, sputtering yield and spectrum/ have been the interest of study over the past two decades or so. These works, as is discussed in a number of publications /Williams 1978a, Winterbon 1977, Sigmund 1969, Lewins 1965/ use either the forward or the backward forms of the pertinent transport equation, and the choice is based either on the nature of the problem or can be quite accidental.

Basically, there are two arguments on which expediency of a particular form can be decided upon. One is the fact that in the forward and backward equations the final and initial variables are operated upon respectively, and thus one of the forms may be superior to the other because of the mathematical consequences of this. With energy dependent scattering and inhomogeneous media this even applies to the Green's function of the problem, which otherwise satisfies both the forward and backward equations. Second, integrals of the Green's function with respect to the final variables satisfy the backward equation only and vica versa, which gives another hint which form is more convenient to use in analogy to neutron transport theory where selection between the two forms of the transport equation is motivated by whether it is the source term or the detector position which varies throughout a series of problems. 
In this paper we choose the direct equation for the collision densities in a multi-species medium. Electronic stopping is neglected right from the beginning. Since we will assume one incident particle, thus representing a point source, the collision density so obtained is essentially the Green's function of the problem. Advantages of the forward equation in connection with heterogeneous media have already been pointed out by williams /1979/. In the particular case presented here, i.e. surface source with a half-space, forward equations are easier to formulate since in the backward equations, source position is operated upon and has therefore to be left arbitrary /Pázsit 1981./. Another advantage of the forward form here is that different quantities of radiation damage will be obtained on integration of the same quantity with respect to the corresponding arguments.

Multi-species collision processes have been the subject of several communications. These works are either essentially space-independent /Kostin 1966, Williams 1976a, 1978b/, or deal with the space dependence by an expansion of the flux or collision density according to Legendre polinomials and spatial moments in infinite media /Dederichs 1965, Winterbon 1980a,b/.

Here, to preserve the possibility of obtaining an analytical result, we approximate the scattering term by the so-called forward-backward model of Fermi in the transport equation sys- 
tem which will enable exact solutions to be obtained for the collision densities of the different species throughout the whole spatial and energy range. Calculation of the collision densities is followed by the determination of the defect distributions and the sputtering spectra. These latter will be given numerically in case of an alien particle being incident on a biatomic target to serve as an illustration of the general theory.

\section{GENERAL THEORY}

If we consider a semi-infinite medium, stretching to the right from the origin, consisting of different species $i=1,2, \ldots N$, into which energetic particles are introduced according to the source functions $Q_{i} / \underset{\sim}{r}, \Omega, E / / i=0, \ldots N /$, the collision density of particles of type $i, G_{i} / \underset{\sim}{r}, \Omega, E /$, will satisfy the forwardtype transport equation system:

$$
\begin{aligned}
& \Omega \nabla_{\sim} \frac{1}{\sigma_{i}(r, E)} G_{i}(\underset{\sim}{r}, \Omega, E)+G_{i}(\underset{\sim}{r}, \Omega, E)=P_{i}(E) \int d \Omega^{\prime} d E^{\prime}\left\{\sum_{j=0}^{N} C_{j i}\left(\underline{r} ; \Omega_{\Omega}^{\prime}, E^{\prime} \rightarrow \Omega, E\right)\right. \\
& \left.\times G_{i j}\left(r, \Omega^{\prime} E^{\prime}\right)+\sum_{j=0}^{N} \tilde{C}_{i j}\left(r ; \Omega^{\prime}, E^{\prime} \rightarrow \Omega, E\right) G_{i}\left(r, \Omega^{\prime}, E^{\prime}\right)\right\}+Q_{i}(\underline{r}, \Omega, E)
\end{aligned}
$$

with the boundary conditions

$$
G_{i}\left({\underset{\sim}{B},}_{\sim}, \underline{\sim}, \sigma\right)=Q_{i}\left(\underline{\sim}_{B}, \Omega, E\right) \sigma_{i}\left(\underline{r}_{B}, E\right) \text { for } \Omega_{x}>0 \text {. }
$$

Although the meaning of the symbols is familiar from literature, let us elaborate a little for later convenience. So $\mathrm{P}_{i} / \mathrm{E} / \mathrm{stands}$ for the damage model. i.e. gives the probability that an atom 
of type i, emerging from a collision with energy $E$ will leave the site. Here we employ the damage model of Kinchin and Pease /1955/, according to which these probabilities are step functions, that is

$$
\begin{aligned}
& \mathrm{P}_{i} / \mathrm{E} /=1 \text { if } \quad \mathrm{E}>\mathrm{E}_{i} \\
& =0 \text { if } E<E_{i} \text {, }
\end{aligned}
$$

where $E_{i}$ is called the displacement energy of the atom of type $i$.

$\mathrm{C}_{j i}$ is the probability density that a particle of type $i$ with $\Omega$ and $E$ will be knocked out by a particle of type $j$ that enters a collision at $\underset{\sim}{\sim}$ with $\Omega^{\prime}$ and $E^{\prime}$.

$\tilde{c}_{i j}$ is the probability density that a particle of type $i$, entering a collision at $\underset{\sim}{r}$ with $\Omega^{\prime}, E^{\prime}$ with an atom of type $j$ will emerge with $\Omega, E$.

It is to be seen that the above definitions imply a recoll at each collision. The fact that a particle can leave the collision site only if its energy is greater than the displacement energy is accounted for by the function $\mathrm{P}_{i} / \mathrm{E} /$ above.

In general, $C_{j i}$ and $\widetilde{C}_{i j}$ can be factorized into terms describing the reaction probabilities, the post-collision energy distributions and the dependence of scattering angle on energy loss, i.e.

$$
C_{j i}\left(\underset{\sim}{r}, \Omega_{n}^{\prime}, E^{\prime} \rightarrow \Omega, E\right)=C_{j i}\left(r, E^{\prime}\right) q_{j i}\left(E^{\prime} \rightarrow E\right) f_{j i}\left(\mu_{0}, E^{\prime} \rightarrow E\right),
$$


and

$$
\tilde{C}_{i j}\left(\underset{\sim}{r} ; \Omega^{\prime}, E^{\prime} \rightarrow \Omega, E\right)=C_{i j}\left(\underset{\sim}{r}, E^{\prime}\right) \tilde{q}_{i j}\left(E^{\prime} \rightarrow E\right) \tilde{f}_{i j}\left(\mu_{0}, E^{\prime} \rightarrow E\right)
$$

where

$$
\mu_{0}=\Omega \Omega^{\prime}
$$

According to the physical meaning of the factors in the collision kernel we have the following normalizations

$$
\sum_{i=0}^{N} c_{j i}(\underset{\sim}{r}, E)=1
$$

for the reaction probabilities,

$$
\int d E q_{j i}\left(E^{\prime} \rightarrow E\right)=\int d E \tilde{q}_{i j}\left(E^{\prime} \rightarrow E\right)=1
$$

for the post-collision energy distributions, and

$$
\int d \Omega f_{j i}\left(\mu_{0}, E^{\prime} \rightarrow E\right)=\int d \Omega \tilde{f}_{i j}\left(\mu_{0}, E^{\prime} \rightarrow E\right)=1
$$

for the scattering angle distribution. Furthermore, energy conservation in a scattering implies the following relation:

$$
\tilde{q}_{i j}\left(E^{\prime} \rightarrow E\right)=q_{i j}\left(E^{\prime} \rightarrow E^{\prime}-E\right) \text {. }
$$

More details on the factorization and functional form of the scattering kernel are given by Williams/1976b/. E.g. for hard spere scattering

$$
\begin{aligned}
& q_{j i}\left(E^{\prime} \rightarrow E\right)=\left\{\begin{array}{cc}
{\left[\left(1-\alpha_{i j}\right) E^{\prime}\right]^{-1}} & \text { for } 0 \leq E \leq\left(1-\alpha_{i j}\right) E^{\prime} ; E^{\prime}<E . \\
0 & \text { otherwise },
\end{array}\right. \\
& \ddot{q}_{i j}\left(E^{\prime} \rightarrow E\right)=\left\{\begin{array}{lr}
{\left[\left(1-\alpha_{i j}\right) E^{\prime}\right]^{-1}} & \text { for } \alpha_{i j} E^{\prime} \leq E<E^{\prime} \sigma E_{0} \\
0 & \text { otherwise },
\end{array}\right.
\end{aligned}
$$

where $\mathrm{E}_{\mathrm{O}}$ is the upper limit on the energy of source functions, and

$$
\alpha_{i j}=\left(\frac{A_{i}-A_{j}}{A_{i}+A_{j}}\right)^{2}
$$

$A_{i}$ being the mass of particles of type $i$. 
As noted before, quantities characteristic of radiation damage are given as integrals of the collision density in the present approach. Before proceeding to the solution of eqns. /1/ we list them first. We define then the following quantities: The number density of particles of type i leaving the halfspace at the surface point $r_{\mathrm{B}}$ in direction $\Omega$ and with energy $E$ is the sputtering distribution, denoted by $s_{i} / r_{B^{\prime}} \Omega, E /$ and is given as

$$
S_{i}\left(\sim_{B}, \Omega, E\right)=\underset{\sim}{n} \Omega G_{i}\left(\sim_{B}, \Omega, E\right) / \sigma_{i}\left(\sim_{B}, E\right) ; \quad \Omega \times<0
$$

where $\underset{\sim}{\mathrm{n}}$ is the outer normal of the half-space at the surface point $\underset{\sim}{\sim}$ B

If a particle of type $i$ is knocked out from its site, irrespective of whether the projectile leaves the site or not, we call it a vacancy of type $i$ and its spatial distribution is given by

$$
V_{i}(\underset{\sim}{r})=\sum_{j=0}^{N} \int d \Omega 2 d E^{\prime} V_{j i}\left(\underline{r}, E^{\prime}\right) G_{j}\left(\underline{r}, \Omega^{\prime}, E^{\prime}\right)
$$

where $v_{j i} / r, E^{\prime} /$ is the probability that a particle of type $j$ with energy $E^{\prime}$ produces a vacancy of type $i$ and is given as

$$
V_{j i}\left(r, E^{\prime}\right)=c_{j i}\left(r, E^{\prime}\right) \int d E P_{i}(E) O_{j i}\left(E^{\prime}-s\right)
$$

Note that $v_{i}$ is neither the distribution of vacant sites since the projectile may occupy it after collision, nor the distribution of sites from where a particle of type $i$ was removed, for the /possibly trapped/ projectile can be of the same type. 
Similarly, if a particle of type $i$ gets stuck in a collision with a particle of species $j$ /irrespective of whether or not the target will recoil/, we call it an interstitial of type ij. The expected number of interstitials at $\underset{\sim}{\sim}$ reads as

$$
I_{i j}(\underline{r})=\int d \Omega d E^{\prime} W_{i j}\left(\underline{r}, E^{\prime}\right) G_{i}\left(r, \Omega, E^{\prime}\right),
$$

where $w_{i j} / \underset{\sim}{r}, \mathrm{E} /$ is the probability that a particle of type $i$ with energy E gets stuck at $\underset{r}{r}$ in a collision with another one of type $j$ :

$$
W_{i j}\left(\underline{r}, E^{\prime}\right)=C_{i j}\left(\underline{r}, E^{\prime}\right) \int d E\left[1-P_{i}(E)\right] \tilde{q}_{i j}\left(E^{\prime} \rightarrow E\right) \text {. }
$$

Finally, if a projectile of type $i$ has insufficient energy to leave the site and it causes a recoil of a particle of type $j$, we call it a replacement. Again, replacements by a particle of the same type are included. The replacement distribution is defined as

$$
R_{i j}(\underline{r})=\int d \Omega_{\sim} d E^{\prime} X_{i j}\left(\underline{r}, E^{\prime}\right) G_{i}\left(\underline{r}, \Omega\left(E^{\prime}\right)\right.
$$

where again, $X_{i j}$ is the probability of a single event, i.e.

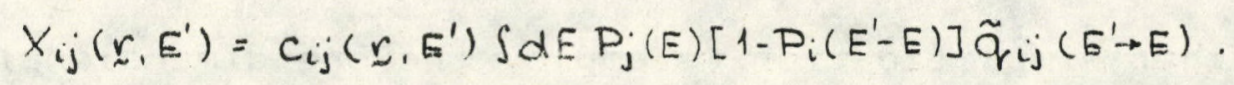

In view of the above,

$$
V_{i}^{*}(r)=V_{i}(r)-\sum_{j=0}^{N} R_{j i}(\underset{\sim}{r})
$$

and

$$
I_{i j}^{*}(r)=I_{i j}(r)-R_{i j}(r)
$$


describe the distributions of the vacant and doubly occupied sites, respectively.

It is stressed that the above relations are based on the assumption that the probability of nonlinear effects / such as the occupying of a vacant site by a recoil etc./ is negligible.

We note finally that particle conservation implies the following relationship between these quantities

$$
\begin{aligned}
& \int d \underset{\sim}{r} d \Omega d E Q_{i}(\underset{\sim}{\sim}, \Omega, E)-\int d{\underset{\sim}{P},}_{2} d \Omega d E S i\left(r_{B}, \Omega, E\right)= \\
& \int d r\left[\sum_{j=0}^{N} I_{i j}^{*}(\underset{\sim}{r}) \cdots V_{i}^{*}(\underset{\sim}{j})\right]
\end{aligned}
$$

3. CALCUlation OF THE COLLision DENSities

The equation system /1/ poses the same mathematical difficulties as those one faces in the course of solution of the single species case. Here, therefore, we have recourse to one of the usual approximations, the synthetic scattering kernels in which the energy and angular dependence is decoupled, although particles are assumed to suffer the appropriate energy loss. For further simplification we employ the one dimensional model of Fermi, according to which particles travel along a straight line but can reverse the direction of travelling with a given probability on collision. Furthermore, energy independent cross sections will be used. The host medium will consist of $\mathrm{N}$ different species, but is assumed to be homogeneous and stretching from $x=0$ to $\Leftrightarrow$ 
In terms of the transport equation, the above assumptions Imply that

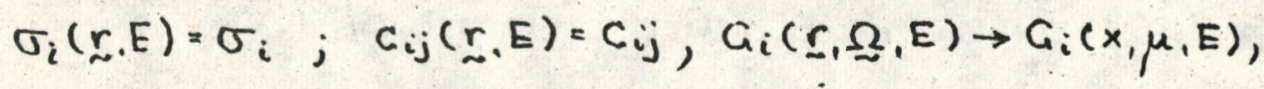

$$
\begin{aligned}
& f_{i j}\left(\mu_{0}, E^{\prime} \rightarrow E\right)=b_{i j} \delta\left(\mu-\mu^{\prime}\right)+\left(1-b_{i j}\right) \delta\left(\mu+\mu^{\prime}\right) \text {, } \\
& \tilde{f}_{i j}\left(\mu_{0}, E^{\prime} \rightarrow E\right)=\tilde{b}_{i j} \delta\left(\mu-\mu^{\prime}\right)+\left(1-\tilde{b}_{i j}\right) \delta\left(\mu+\mu^{\prime}\right) \text {, }
\end{aligned}
$$

where $\mu$ and $\mu^{\prime}$ are the pre- and post-collision directions of flight, respectively, and they can only take the values \pm 1 .

Finally, assume that the post collision energy distributions depend on $E^{\prime} / E$ as

$$
q_{i j}\left(E^{\prime} \rightarrow E\right)=\frac{1}{E^{\prime}} h_{i j}\left(E^{\prime} / E\right),
$$

and

$$
\tilde{q}_{i j}\left(E^{\prime} \rightarrow E\right)=\frac{1}{E^{\prime}} \tilde{h}_{i j}\left(E^{\prime} / E\right) \text {. }
$$

We shall assume one incoming particle of type 0 , being incident on the free surface, which means that the source term takes the form

$$
Q_{i}(x, \mu, E)=\delta_{i, 0} \delta(x) \delta(\mu-1) \delta\left(E-E_{0}\right),
$$

and will enter the equations in form of a boundary condition only .

Introducing the lethargy variable $u=\ln \left(E_{O} / E\right)$ and allowing for $|10|$ and $|11|$, eqns $|1|$ reduce to 
$\left(\frac{\mu}{\sigma_{i}} \frac{\partial}{\partial x}+1\right) G_{i}(x, \mu, u)=P_{i}(E) \int_{0}^{u} d u^{i}\left\{\sum_{j=0}^{N} c_{j i} h_{j i}\left(u-u^{i}\right)\left[b_{j i} C_{j}\left(x, 1, u^{\prime}\right)\right.\right.$

$\left.\left.+\left(1-b_{j i}\right) G_{j}\left(x,-1, u^{\prime}\right)\right]+\sum_{j=0}^{N} c_{i j} \tilde{h}_{i j}\left(u-u^{\prime}\right)\left[\tilde{b}_{i j} G_{i}\left(x, 1, u^{\prime}\right)+\left(1-\tilde{b}_{i j}\right) G_{i}\left(x_{1}-1, u^{\prime}\right)\right]\right\}$,

with the boundary conditions

and

$$
\lim _{x \rightarrow \infty} G_{i}(x, \mu, u)=0 \text {, }
$$

$$
G_{i}(0,1, u)=\delta_{i, 0} \delta(u) \sigma_{0} / E_{0} \text {. }
$$

$G_{i} / x, \mu, u /$ here denotes the collision density per unit

energy interval. We shall now assume that the displacement energies $E_{1}$ are all equal to $E_{d}$, in which case one can formally omit $P_{1} / E /$ in $/ 12 /$ on the understanding that $G_{1} / x, \mu, E /$ is the true solution for $E>E_{d}$ whilst $G_{1}=0$ for $E<E_{d}$.

Taking Laplace transform with respect to u yields

$\left(\frac{\mu}{\sigma_{i}} \frac{\partial}{\partial x}+1\right) \bar{G}_{i}(x, \mu, s)=\sum_{j=0}^{N} \lambda_{j i}(s)\left[b_{j i} \bar{G}_{j}(x, 1, s)+(1-1, j j i) \bar{G}_{j}(x,-1, s)\right]$

$+\sum_{j=0}^{N} \tilde{\lambda}_{i j}(s)\left[\tilde{b}_{i j} \bar{G}_{i}(x, 1,3)+\left(1-\tilde{b}_{i j}\right) \bar{G}_{i}(x,-1, s)\right]$,

where

$$
\lambda_{j i}(s)=c_{j i} \bar{h}_{j i}(s) ; \quad \tilde{\lambda}_{i j}(s)=c_{i j} \overline{\widetilde{h}}_{i j}(s) .
$$

The boundary conditions read

$$
\begin{aligned}
& \lim _{x \rightarrow \infty} \bar{G}_{i}(x, \mu, \sigma)=0, \\
& \bar{G}_{i}(0,1, s)=\delta_{i, 0} \sigma_{0} / E_{0} .
\end{aligned}
$$

Introducing the functions

$$
\bar{G}_{i}(x, s)=\bar{G}_{i}(x, 1, s)+\bar{G}_{i}(x,-1, s),
$$

and

$$
\bar{g}_{i}(x, s)=\bar{G}_{i}(x, 1, s)-\bar{G}_{i}(x,-1, s) \text {. }
$$


leads to

$$
\frac{1}{\sigma_{i}} \frac{\partial}{\partial x} \bar{G}_{i}(x, s)=\sum_{j=0}^{N} a_{i j} \bar{g}_{j}(x, s),
$$

and

$$
\frac{1}{\sigma_{i}} \frac{\partial}{\partial x} \bar{g}_{i}(x, s)=\sum_{j=0}^{N} d_{i j} \bar{G}_{j}(x, s),
$$

where

$$
\begin{aligned}
& \alpha_{i j}=\lambda_{j i}\left(2 b_{j i}-1\right)+\delta_{i j}\left[\sum_{k=0}^{N} \tilde{\lambda}_{i k}\left(2 \tilde{b}_{i k}-1\right)-1\right], \\
& d_{i j}=\lambda_{j i}+\delta_{i j}\left[\sum_{k=0}^{N} \tilde{\lambda}_{i k}-1\right] .
\end{aligned}
$$

Note that for isotropic scattering, 1 .e. if $b_{1 j}=\tilde{b}_{j 1}=1 / 2$ one has $a_{i j}=-\delta_{i j}$.

Differentiating the first eqn in $/ 15 /$ will help eliminate $\bar{g}_{1}$ to arrive at

$$
\frac{1}{\sigma_{i}^{2}} \frac{\partial^{2}}{\partial x^{2}} \bar{G}_{i}(x, s)=\sum_{j=0}^{N} t_{i j}(s) \bar{G}_{j}(x, s),
$$

with

$$
t_{i j}=\sum_{k=0}^{N} a_{i k} d_{k j} \frac{\sigma_{k}}{\sigma_{i}}
$$

In case of isotropic scattering $t_{i j}=-d_{i j}$.

Making use of the relation

$$
\bar{g}_{i}(0,5)=2 \bar{G}_{i}(0,1,5)-\bar{G}_{i}(0,5) \text {, }
$$

the boundary condition can be inverted to

$$
\frac{1}{\sigma_{i}} \frac{\partial}{\partial x} \bar{G}_{i}(0, s)=\frac{2}{E_{0}} \delta_{i, 0} a_{00} \sigma_{0}-\sum_{j=0}^{N} a_{i j} \bar{G}_{j}(0, s) .
$$

The general solution of equation system $/ 16 /$ is

$$
\bar{G}_{i}(x, s)=\sum_{n=0}^{N} \varphi_{n}^{(i)}(x) e^{-\nu_{n} x}
$$

where $\varphi_{n} / 1 /|x|$ are polynomials and the $\nu_{n}$ are the positive roots of the characteristic equation 


$$
\operatorname{det}\left[t_{i j}-\delta_{i j} \frac{\nu^{2}}{\sigma_{i}^{2}}\right]=0 \text {. }
$$

Assuming for the moment that the characteristic equation has $n+1$ nondegenerate roots with positive real parts, the coefficients $\varphi_{n}^{(i)}$ are constants and as seen from eqn $\mid 16 /$, they satisfy the equation system

$$
\frac{\nu_{n}^{2}}{\sigma_{i}^{2}} \varphi_{n}^{(i)}=\sum_{j=0}^{N} t_{i j} \varphi_{n}^{(j)}
$$

$\overline{\mathrm{G}}_{i} \rightarrow \circ$ for $\mathrm{x} \rightarrow \infty$ can be ensured by assuming $\operatorname{Re}\left|\nu_{n}\right|>0$ if $\operatorname{Re} / \mathbf{s} />s_{0}$ with some given $s_{0}$, and it follows from eqn $/ 17 /$ that

$$
\sum_{n=0}^{N} \frac{\nu_{n}}{\sigma_{i}} \varphi_{n}^{(i)}=\frac{2}{E_{0}} \delta_{i, 0} \alpha_{00} \sigma_{0}-\sum_{j=0}^{M} \alpha_{i j} \sum_{n=0}^{N} \varphi_{n}^{(j)} \text {. }
$$

It may be worth noting that for sufficiently large real values of $\mathrm{s}$

$$
\lambda_{j i} \approx \tilde{\lambda}_{i j} \ll 1 \text {, }
$$

both $\lambda_{j i}$ and $\tilde{\lambda}_{i j}$ being proportional to Laplace transformed functions, thus

$$
\alpha_{i j} \approx \alpha_{i j} \approx-\delta_{i j},
$$

i.e.

$$
t_{i j} \approx \delta_{i j} \text {. }
$$

Consequently for large values of $s$ the roots of the characteristic equation lie near the $\sigma_{i}-s$ :

$$
\nu_{i} \approx \sigma_{i}
$$

and thus if the cross sections $\sigma_{i}$ are all different the characteristic equation is expected to have $n+1$ different roots with positive real parts. In the next section explicit bounds on the roots will be given for the case of isotropic hard-sphere scattering . 
According to the above results the Laplace transforms of the collision densities can be determined simply by solving a number of algebraic equations. The inverse transform is, however a rather invidious task and can only be performed in some special cases. A couple of examples when the analytical inversion is possible is reported by Lux and Pázsit/1981/ for the single species case. In more complicated cases one of the numerical methods is to be resorted to.

In the next section, the case of hard-sphere isotropic scattering is examined in some depth. Extension of the results to energy dependent cross sections is in progress.

\section{THE CASE OF ISOTROPIC HARD SPHERE SCATTERING}

In what follows it will be assumed that the scattering is isotropic in the L.S. and that the post collision energy is distributed uniformly in an interval determined by the mechanical hard sphere law. This means that $q_{j i}$ and $\tilde{q}_{i j}$ are given by $/ 2 /$, and also that

$$
\begin{aligned}
& h_{i j}=\left\{\begin{array}{cl}
\left(1-\alpha_{i j}\right)^{-1} & \text { for } 0 \leq E \leq\left(1-\alpha_{i j}\right) E^{\prime}, \\
0 & \text { otherwise },
\end{array} E^{\prime} \leq E_{0}\right. \\
& \tilde{h}_{i j}=\left\{\begin{array}{cc}
\left(1-\alpha_{i j}\right)^{-1} & \text { for } \alpha_{i j} E^{\prime} \leq E \leq E^{\prime} \leq E_{0} \\
0 & \text { otherwise }
\end{array}\right.
\end{aligned}
$$

in eqn $/ 11 ;$ and onwards. $\lambda_{j i} / s /$ and $\tilde{\lambda}_{i j} / \mathrm{s} /$ in eqn $/ 13 /$ will read

$$
\lambda_{j i}(s)=\frac{C_{j i}}{s}\left(1-\alpha_{i j}\right)^{s-1}, \tilde{\lambda}_{i j}(s)=\frac{C_{i j}}{s} \frac{1-\alpha_{i j}^{s}}{1-\alpha_{i j}} .
$$


Recalling that $\alpha_{i j}=-\delta_{i j}$ in the isotropic case,

$$
t_{i j}=-\alpha_{i j}=-\lambda_{j i}-\delta_{i j}\left(\Lambda_{i}-1\right) \text {, }
$$

where

$$
\Lambda_{i} \cdot \sum_{k=0}^{N} \tilde{\lambda}_{i k}
$$

In this simplified case the differential equation system /16/ becomes

$$
\left(1-\Lambda_{i}-\frac{1}{\sigma_{i}^{2}} \frac{\partial^{2}}{\partial x^{2}}\right) \bar{G}_{i}(x, s)=\sum_{j=0}^{N} \lambda_{j i} \bar{G}_{j}(x, s),
$$

and the algebraic equations $/ 19 /$ and $/ 20 /$ reduce to

$\left(\frac{\nu_{n}^{2}}{\sigma_{i}^{2}}+\Lambda_{i}-1\right) \varphi_{n}^{(i)}+\sum_{j=0}^{N} \lambda_{j i} \varphi_{n}^{(j)}=0$

$$
1=0,1, \ldots . . N ; n=0,1, \ldots . .
$$

and

$\sum_{n=0}^{N}\left(\frac{\nu_{n}}{\sigma_{i}}+1\right) \varphi_{n}^{(i)}=\frac{2}{E_{0}} \delta_{i, 0} \sigma_{0}$

$, 1=0,1, \ldots n$

where the $\nu_{n}^{2}-s$ are the roots of the characteristic equation

$$
\operatorname{det}\left[\lambda_{j i}+\delta_{i j}\left(\Lambda_{i}-1+\frac{\nu^{2}}{\sigma_{i}^{2}}\right)\right]=0 \text {, }
$$

and the $\nu_{n}-s$ are chosen as the branches of the square roots that have positive real parts for $\mathrm{Re} / \mathrm{s} /$ being sufficiently large. As it is shown in the Appendix, the roots of the characteristic equation $/ 26 /$ satisfy the inequalities

$$
\left|\frac{\bar{\nu}_{i}^{2}}{\sigma_{i}^{2}}-1\right| \leq \frac{2}{|s|}\left(1+\sum_{j=1}^{N} c_{i j} \frac{\alpha_{i j}^{2}}{1-\alpha_{i j}}\right) \text { if } \operatorname{Re}(s) \geqslant 2 \text {. }
$$

These inequalities facilitate the determination of the roots by confining them into a circle around $\sigma_{i}^{2}$, but they also amount to saying that $\operatorname{Re} / \nu_{i} />0$ if 
$|s| \geq 2\left(1+\sum_{j=1}^{N} c_{i j} \frac{\alpha_{i j}^{2}}{1-\alpha_{i j}}\right)$ and $\operatorname{Re}(s) \geq 2$.

The latter result enables one to select the most suitable path of integration in numerical inverse Laplace transformations.

\section{4.a. Defect distributions}

In the isotropic hard sphere scattering model eqn /5/

\section{becomes}

$V_{j i}\left(x, E^{\prime}\right)=C_{j i} \int_{E_{d}}^{\left(1-\alpha_{i j}\right) E^{\prime}} d E\left[E^{\prime}\left(1-\alpha_{i j}\right)\right]^{-1}=\frac{C_{j i}}{1-\alpha_{i j}}\left(1-\alpha_{i j}-\frac{E_{d}}{E^{\prime}}\right)$ if $\left(1-\alpha_{i j}\right) E^{\prime} \geq E_{d}$,

and passing on to the lethargy variable, the vacancy distribution from $/ 4 /$ reads

$V_{i}\left(x, u_{d}\right)=\sum_{j=0}^{N} \frac{E_{0} C_{j i}}{1-\alpha_{i j}} \int_{0}^{u_{i j}} d u\left[\left(1-\alpha_{i j}\right) e^{-u}-e^{-u_{d}}\right] G_{j}(x, u)$,

where

$$
u_{d}=\ln \left(E_{0} / E_{d}\right) ; \quad u_{i j}^{+}=\max \left(0, u_{d}+\ln \left(1-\alpha_{i j}\right)\right) .
$$

Its Laplace transform with respect to $u_{d} 1 s$

$$
\begin{aligned}
\bar{V}_{i}(x, s) & =E_{0} \sum_{j=c}^{N} c_{j i}\left(1-\alpha_{i j}\right)^{s}\left[\frac{1}{s}-\frac{1}{s+1}\right] \bar{G}_{j}(x, s+1)= \\
& =\frac{E_{0}}{s} \sum_{j=0}^{N} \lambda_{j i}(s+1) \bar{G}_{j}(x, s+1),
\end{aligned}
$$

or from eqn $/ 23 /$

$$
\bar{v}_{i}(x, s)=\frac{E_{0}}{s}\left[1-\Lambda_{i}(s+1)-\frac{1}{\sigma_{i}^{2}} \frac{\partial^{2}}{\partial x^{2}}\right] \bar{G}_{i}(x, s+1) \text {. }
$$

Similarly, from eqns $/ 6 /$ and $/ 7 /$ for the interstitial distributions we have

$$
I_{i j}\left(x, u_{d}\right)=\frac{E_{0} C_{i j}}{1-\alpha_{i j}} \int_{u_{i j}^{-}}^{u_{d}} d u\left(e^{-u_{d}}-\alpha_{i j} e^{-u}\right) G_{i}(x, u),
$$

with $u_{i j}^{-}=\max \left(0, u_{d}+\ln \alpha_{i j}\right)$. 
Taking Laplace transform yields

$$
\bar{I}_{i j}(x, s)=\frac{E_{0} C_{i j}}{s}\left[1-\frac{1-\alpha_{i j}^{s+1}}{(s+1)\left(1-\alpha_{i j}\right)}\right] \bar{G}_{i}(x, s+1)=\frac{E_{0}}{s}\left[C_{i j}-\tilde{\lambda}_{i j}(s+1)\right] \bar{G}_{i}(x, s+1) \text {. }
$$

Iinally for the replacement distributions, from eqns $/ 8 /$ and $/ 9 /$

$$
\begin{aligned}
X_{i j}\left(x, E^{\prime}\right) & =\int_{\max \left(E_{d}, E^{\prime}-E_{d}\right)}^{\left(1-\alpha_{i j}\right) E^{\prime}} d E c_{i j}\left[\left(1-\alpha_{i j}\right) E^{\prime}\right]^{-1}= \\
& = \begin{cases}\frac{c_{i j}}{1-\alpha_{i j}}\left[\frac{E_{d}}{E^{\prime}}-\alpha_{i j}\right] & \text { if } 2 E_{d} \leq E^{\prime} \leq \min \left(E_{0}, \frac{E_{d}}{\alpha_{i j}}\right) \\
\frac{c_{i j}}{1-\alpha_{i j}}\left[\left(1-\alpha_{i j}\right)-\frac{E_{d}}{E^{\prime}}\right] & \text { if } \frac{E_{d}}{1-\alpha_{i j}} \leq E^{\prime} \leq \min \left(E_{0}, 2 E_{d}\right) .\end{cases}
\end{aligned}
$$

Thus, in terms of lethargy the replacement distribution takes the form

$$
\begin{aligned}
R_{i j}\left(x, u_{d}\right) & =\frac{E_{0} c_{i j}}{1-\alpha_{i j}}\left\{\int_{u_{i j}^{-}}^{u_{d} \cdot \ln 2} d u\left(e^{-u_{d}}-\alpha_{i j} e^{-u}\right) G_{i}(x, u)+\right. \\
& \left.+\int_{u_{d} \cdot \ln 2}^{u_{i j}^{+}} d u\left[\left(1-\alpha_{i j}\right) e^{-u}-e^{-u_{d}}\right] G_{i}(x, u)\right\},
\end{aligned}
$$

noting that the integrals are to be set to zero whenever the lower limits of integrations exceed the upper ones. Practically that means that the first integral is nonzero if

$$
2 E_{d}<E_{d} / \alpha_{i j},
$$

and the second is nonzero if

$$
E_{d} /\left(1-\alpha_{i j}\right)<2 E_{d} \text {, }
$$

the two conditions leading to the conclusion that there is no replacement if

$$
\alpha_{i j}>0.5
$$

Accordingly, for $\alpha_{i j}>0.5 \mathrm{~V}_{i}$ and $I_{i j}$ do represent the distributions of the vacant and doubly occupied sites.

Taking Laplace transform of the replacement density with respect to $u_{d}$ yields 


$$
\bar{R}_{i j}(x, s)=\frac{E_{0} c_{i j}}{\left(1-\alpha_{i j}\right) s(s+1)}\left[\alpha_{i j}^{s+1}+\left(1-\alpha_{i j}\right)^{s+1}-2^{-s}\right] \bar{G}_{i}(x, s+1)
$$

for $\alpha_{i j}<1 / 2$.

Inversion of these formulae in the single species medium is given by Lux and Pázsit /1981/.

\section{4.b. Sputtering spectra}

Once the collision densities have been obtained, the sputtering distributions per unit lethargy are readily available by $/ 3 /$ and $/ 17 /$ as

$$
S_{i}(u)=E_{0} e^{-u} \cdot G_{i}(0, u) / \sigma_{i}-\delta_{i, 0} \delta(u) \text {, }
$$

or in the Laplace domain

$$
\bar{S}_{i}(s)=E_{0} \bar{G}_{i}(0, s+1)-\delta_{i, 0} \text {. }
$$

According to $/ 18 /$,

$$
\bar{G}_{i}(0,5)=\sum_{n=0}^{N} \varphi_{n}^{(i)} \text {, }
$$

where the $\varphi_{n}^{(i)}$ are the solutions of the algebraic equation systems $/ 19 /$ and $/ 20 /$.

In the isotropic hard-sphere case the simplifications represented in eqns $|21 /-| 26 /$ can be allowed for. In what follows we shall illustrate this by considering the case of an alien particle impinging on a binary target. To take advantage of any possible analogies with the theory of neutron thermalization, the alien particle will sometimes be referred to as a neutron. 
Since the medium does not contain the primary particle $c_{j 0}=\lambda_{j 0}=\tilde{\lambda}_{j 0}=0, \quad \Lambda_{0}=\tilde{\lambda}_{01}+\tilde{\lambda}_{02}$,

which means that the equations of $G_{0}$ decouple from those of $G_{1}$ and $\mathrm{G}_{2}$. Accordingly,

$$
\begin{aligned}
\nu_{0}^{2} & =\sigma_{0}^{2}\left(1-\Lambda_{0}\right) \\
E_{0} \varphi_{0}^{(0)} & =2 \sigma_{0}^{2} /\left(\sigma_{0}+\nu_{0}\right), \quad \varphi_{1}^{(0)}=\varphi_{2}^{(0)}=0,
\end{aligned}
$$

and thus the neutron sputtering spectrum in the Laplace domain is

$$
\bar{s}_{0}(s)=\left[\frac{2 \sigma_{0}}{\sigma_{0}+\nu_{0}}-1\right]=\frac{\sigma_{0}-\nu_{0}(s+1)}{\sigma_{0}+\nu_{0}(s+1)} .
$$

To determine $S_{1}$ and $S_{2}$, first one has

$$
\nu_{1,2}^{2}=\frac{1}{2}\left[d_{1}+d_{2} \pm \sqrt{\left(d_{1}-d_{2}\right)^{2}+4 d}\right]
$$

where

$$
d_{i}=\sigma_{i}^{2}\left(1-\Lambda_{i}-\lambda_{i i}\right), \quad i=1,2,
$$

and

$$
d=\left(\sigma_{1} \sigma_{2}\right)^{2} \lambda_{12} \lambda_{21}
$$

For the $\varphi_{n}^{(i)} \mid i=1,2 /$, eqn $/ 24 /$ yields

$$
\begin{aligned}
\left(\alpha_{1}-\nu_{n}^{2}\right) \varphi_{n}^{(1)}-\sigma_{1}^{2} \lambda_{21} \varphi_{n}^{(2)} & =\sigma_{1}^{2} \lambda_{01} \varphi_{n}^{(0)} \\
& -\sigma_{2}^{2} \varphi_{n}^{(1)}+\left(d_{2}-\nu_{n}^{2}\right) \varphi_{n}^{(2)}=\sigma_{2}^{2} \lambda_{02} \varphi_{n}^{(0)}
\end{aligned}
$$

which, since the r.h.s. is nonzero for $n=0$ only, serve to determine $\varphi_{0}^{(1)}$ and $\varphi_{0}^{(2)}$, while for $n=1$ and 2 they coincide to yield

$$
\varphi_{n}^{(2)}=\left[\left(d_{1}-\nu_{n}^{2}\right) / \sigma_{1}{ }^{2} \lambda_{21}\right] \varphi_{n}^{(1)}
$$


Eventually, the remaining four coefficients $\varphi_{n}^{(i)} / 1, n=1,2 /$ can be obtained by putting $/ 28 /$ into $/ 25 /$ which, taking $\varphi_{0}^{(i)}$ as known can be cast into the form

$$
\begin{aligned}
& \sum_{n=1}^{2}\left(\sigma_{1}+\nu_{n}\right) \varphi_{n}^{(1)}=-\left(\sigma_{1}+\nu_{0}\right) \varphi_{0}^{(1)} \\
& \sum_{n=1}^{2}\left(\sigma_{2}+\nu_{n}\right) \frac{d_{1}-\nu_{n}^{2}}{\sigma_{1}^{2} \lambda_{21}} \varphi_{n}^{(1)}=\left(-\sigma_{2}+\nu_{0}\right) \varphi_{0}^{(2)} .
\end{aligned}
$$

Having determined $\varphi_{n}^{(i)}|n=1,2|$, the remaining spectra are given as

$$
\bar{S}_{i}(s)=E_{0} \sum_{n=0}^{2} \varphi_{n}^{(i)}(s+1) / \sigma_{i}
$$

$\bar{S}_{1}$ and $\bar{S}_{2}$ are in principle similar, though more complicated constructs as $\bar{S}_{0} / s /$. Their inversion is hindered by the fact that the $\nu_{n}^{2}$ will have an infinite number of zeros / Sengupta and Srikantiah, 1974/, leading to an infinity of branch cuts in $\overline{\mathrm{S}}_{i} / \mathrm{s} /$ on the complex s plane /Williams,1979/. Inversion can be given as a sum of line integrals, the one along the cut on the real axis giving the asymptotic solution, and the rest accounting for the Placzek discontinuities /Williams 1979/.

This technique, although theoretically exact, is not very useful for numerical calculation of the spectra as the zeros of $\nu_{n}^{2}$ can only be given approximately /Sengupta and srikantiah 1974/, and also that especially for small u values $\left[u<<\ln \left(1 / \alpha_{01}\right)\right]$ a very large number of terms has to be considered to attain convergence /Williams 1966/. 
Thus, it seems much more expedient to invert $/ 27 /$ and /29/ by direct application of numerical inversion codes. This, as mentioned before, is facilitated by the approximate knowledge of the singularities of $\bar{s}_{i}$ on the complex plane which enables one to select the path of integration in the most effective way from the numerical point of view.

The results of a calculation of $s_{i} / u /$, with the parameters as given in Table $\mathbf{I}$. are displayed in Fig.1. The Placzek discontinuities at $u=-1 n \alpha_{o l}, u=-\ln / 1-\alpha_{o l} /$, etc. can well be noticed. The nature of these discontinuities is investigated in another forthcoming publication.

We also notice that the spectra $S_{1}$ and $S_{2}$ increase with lethargy in contrast with $S_{O^{\prime}}$ due to there being an increasing number of recoils, a fact observed by Williams /1976a/.

5. SUMMARY AND CONCLUSIONS

Introduction of the Fermi scattering model into the forward equations of the multispecies transport problem and Laplace transform with respect to lethargy reduced the problem to a form amenable to analytic solution. Collision densities, defect distributions and sputtering spectra were given in closed form in the Laplace domain. This result in itself is interesting since energy deposition, another characteristics of radiation damage, is proportional to $\overline{\mathrm{G}}_{i} / \mathrm{x}, \mathrm{s}=1 /$ /Williams, 1979 / without 


$$
4
$$


the need to inverting it. Calculation of the defect distributions and sputtering spectra nevertheless necessitates performing the inverse Laplace transform. Analytical inversion of these functions is possible in principle, based on the works of Sengupta and Srikantiah /1974/ and Williams/1979/. The resulting expressions are, however, not especially well suited for analytical work. Some investigation of the analytic properties of the characteristic equation, presented here, made application of numerical inversion codes troublefree. This was illustrated by calculating the sputtering spectra for a biatomic target with an alien incident particle. The results are in agreement with some other work in this field.

The situation occuring here bears some resemblance to other cases of radiation damage calculations. For instance, in three dimensional calculations, space-energy dependent coefficient of the Legendre polinomials are given in the Laplace domain as solutions of a linear equation system /Dederichs 1965, Winterbon 1980 a/. This is just another situation where numerical Laplace transform seems promising, this method is therefore likely to have more applications in radiation damage problems.

\section{APPENDIX}

Bounds for the roots of the characteristic equation Introduce a new quantity $\mu_{i}$ instead of the $i-t h$ root of the characteristic equation $/ 26 /$ as 


$$
\mu_{i}=1-\frac{\nu_{i}^{2}}{\sigma_{i}^{2}},
$$

then the characteristic determinant /after transposition/ takes the form

$$
\operatorname{det}\left[\lambda_{i j}+\delta_{i j}\left(\Lambda_{i}-\mu_{i}\right)\right]=0 \text {. }
$$

According to classical results the eigenvalue $\mu_{1}$ of the determinant falls into the Gersgorin circle

or

$$
\left|\mu_{i}-\Lambda_{i}-\lambda_{i i}\right| \leqslant \sum_{\substack{j=1 \\ j \neq i}}^{N}\left|\lambda_{i j}\right|,
$$

$$
\left|\mu_{i}\right| \leqslant \sum_{j=1}^{N}\left|\lambda_{i j}\right|+\left|\Lambda_{i}\right|
$$

It follows from eq $/ 21 /$ that

$$
\left|\lambda_{i j}\right|=\frac{c_{i j}}{1-\alpha_{i j}}\left|\left(1-\alpha_{i j}\right)\right| / 1 s \mid=\frac{c_{i j}}{|s|}\left(1-\alpha_{i j}\right)^{x-1}
$$

where $x=\operatorname{Re} / s /$. Now for $x>2$

$$
\left|\lambda_{i j}\right| \leqslant \frac{C_{i j}}{|s|}\left(1-\alpha_{i j}\right) \text {, }
$$

and, taking into account that

$$
\sum_{j=0}^{N} c_{i j}=1
$$

we have

$$
\sum_{j=1}^{N}\left|\lambda_{i j}\right| \leq \frac{1}{|s|}\left(1-\sum_{j=1}^{N} C_{i j} \alpha_{i j}\right) \quad \text { for } \operatorname{Re} / s \mid>2
$$

Turning to the second term on the r.h.s. of eqn $/ \mathrm{Al} /$, from eqn $|22|$

and thus

$$
\left|\Lambda_{i}\right|=\frac{1}{|s|} \sum_{j=1}^{N} c_{i j} \frac{\left|1-\alpha_{i j}^{s}\right|}{\mid-\alpha_{i j}} \leq \frac{1}{|s|} \sum_{j=1}^{N} c_{i j} \frac{1+\alpha_{i j}^{x}}{1-\alpha_{i j}},
$$

$$
\left|\Lambda_{i}\right| \leq \frac{1}{15} \mid \sum_{j=1}^{N} c_{i j} \frac{1+\alpha_{i j}{ }^{2}}{1-\alpha_{i j}} \quad \text { for } \operatorname{Re} / \mathrm{s} \mid>2
$$

Insertion of eqs /A2/ and /A3/ into /A1/ yields 


$$
\left|\mu_{i}\right|=\left|\frac{\nu_{i}{ }^{2}}{\sigma_{i}{ }^{2}}-1\right| \leq \frac{2}{\mid s 1}\left(1+\sum_{j=1}^{n} c_{i j} \frac{\alpha_{i j}^{2}}{1-\alpha_{i j}}\right) \quad \text { for } \operatorname{Re} / \mathrm{s} \mid>2 \text {. }
$$

We note that by analogy with the derivation above it can be seen that for real $s$ values

$$
\left|u_{i}\right| \leq \frac{2}{3}
$$

if $\mathrm{s}>2$, real. 
TABLE 1

\begin{tabular}{c|ccl}
$i$ & 0 & 1 & 2 \\
\hline$\alpha_{0 i}$ & 0.0 & 0.4444 & 0.8186 \\
$c_{0 i}$ & 0.0 & 0.5 & 0.5 \\
$c_{1 i}$ & 0.0 & 0.7 & 0.3 \\
$c_{2 i}$ & 0.0 & 0.3 & 0.7 \\
$\sigma_{i}$ & 1.0 & 10. & 3.0 \\
$\alpha_{12}=0.36$ & & &
\end{tabular}


REFERENCES

Dederichs P.H. /1965/ Phys.Stat.Sol.10, 303-18

Kinchin G.H. and Pease R.S. /1955/ Rep.Prog.Phys.18,1

Kostin M.D. /1966/ J.Appl.Phys. 37 3801-4

Lewins J./1965/ Importance, the adjoint equation. Pergamon Press.

Lux I, and Pázsit I. /1981/ Submitted to Rad.Effects.

Pázsit I./1981/ Phys.Stat.Sol.2, Vol.103.

Sengupta A. and Srikantiah G./1974/ J.Phys.D.7, 1918-27

Sigmund P./1969/ Phys. Rev. 184,383

Williams M.M.R. /1966/ The slowing down and thermalisation of neutrons. /Amsterdam:North-Holland/

$\begin{array}{ll}- & \text { /1976a/ Rad.Effects 30, 147-57 } \\ - & 11976 \mathrm{~b} / \mathrm{J} . \text { Phys.A.9, } 771-83 \\ - & 11978 \mathrm{a} / \mathrm{Ann} . \mathrm{Nucl.Energy} 5,149-50 \\ - & 11978 \mathrm{~b} / \text { Rad.Effects } 38,159-64 \\ - & 11979 / \text { Ann.Nucl.Energy } \underset{\sim}{6}, 154-73\end{array}$

Winterbon K.B. /1977/ Appl.Phys.Lett. 31, 649

/1980a/ Rad.Effects $46,181-88$

/1980b/ Rad.Effects 48, 97-100 



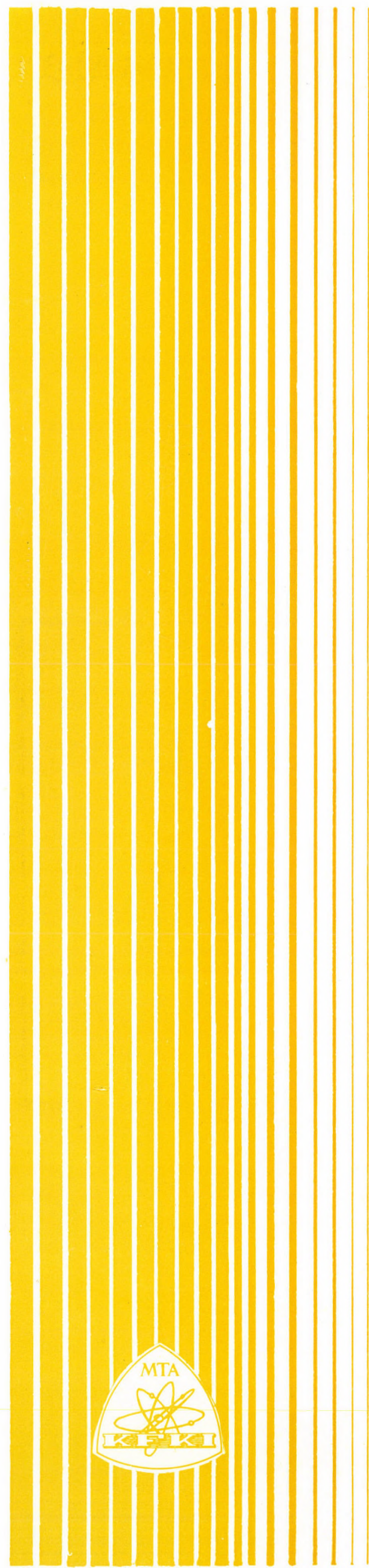

Kiadja a Központi Fizikai Kutató Intézet Felelôs kiadó: Gyimesi Zoltán

Szakmai lektor: Szatmáry Zoltán Nyelvi lektor: Valkó János

példányszám: 310 Törzsszám: 81-304 Készült a KFKI sokszorositó üzemében Felelốs vezetõ: Nagy Károly

Budapest, 1981. május hó 